Received: 2016.12 .23

Accepted: 2017.01 .26

Published: 2017.10.20

Authors' Contribution:
A Study Design
B Data Collection
C Statistical Analysis
D Data Interpretation
E Manuscript Preparation
F Literature Search
G Funds Collection

Material/Methods:

Results:

Conclusions:

MeSH Keywords:

PDF file:

\section{Accuracy of Ultrasonography (US) and Magnetic Resonance Imaging (MRI) in Detection of Rotator Cuff Tears in District General Hospital}

\author{
Ahmed Elmorsy ${ }^{\mathrm{ABCDEF}}$, Andrew Keightley ${ }^{\text {BE, }}$, Mark Flannery ${ }^{\mathrm{ACDEF}}$ \\ Department of Trauma and Orthopaedics, Royal Surrey County Hospital, Guildford, U.K. \\ Author's address: Ahmed Elmorsy, Royal Surrey County Hospital, Egerton road, Guildford, GU2 7XX, U.K., \\ e-mail: ahmedelmorsy@doctors.org.uk
}

\section{Summary}

Rotator cuff tears (RCTs) represent a significant proportion of shoulder diseases, hence they are a frequent cause of patient visits in shoulder clinics. However, the diagnosis of rotator cuff tears is controversial. Investigation of cuff tears is based on ultrasonography (US) and magnetic resonance imaging (MRI). Both modalities have been in use for decades, and their advantages and limitations are known. A recent Cochrane review of the subject suggested that US and MRI both performed well with respect to full thickness rotator cuff tears (FTT). However, they were less accurate with respect to partial thickness tears (PTT). The aim of this study is to assess the accuracy of US and MRI in diagnosing rotator cuff tears.

This is a retrospective analysis of a cohort of 255 patients who underwent shoulder arthroscopy. Of them, 125 patients had preoperative US, and 130 had preoperative MRI. The imaging results were compared with arthroscopic findings for patient.

After calculating sensitivity, specificity, positive prediction value (PPV), and negative prediction value, we found no statistically significant difference between US and MRI in detection of rotator cuff tears of any type (RCT) or FTT. However, US is more specific in detecting PTT compared to MRI $(\mathrm{P}=0.00008)$ but with no significant difference in other parameters.

We concluded that US and MRI both have similar accuracy in diagnosing RCT of any sort and FTT However, US is more specific than MRI in detecting PTT. In our institute, we now recommend US as the investigation of choice for diagnosing rotator cuff tears.

\section{Magnetic Resonance Imaging, Interventional • Rotator Cuff • Shoulder Impingement Syndrome •} Ultrasonography

http://www.polradiol.com/abstract/index/idArt/903004

\section{Background}

Rotator cuff tears are responsible for a significant proportion of shoulder pain cases, hence they are a frequent cause of patient visits in shoulder clinics. However, their diagnosis and definitive management has been a cause of controversy. Shoulder pain can be a vague symptom, and examination can be inconclusive in decision making.

Cuff tear investigation is based on non-invasive radiography with the use of ultrasonography (US) and magnetic resonance imaging (MRI). Ultrasound has been used for decades for the diagnosis of shoulder pathology. It is relatively cheap, acceptable to patients, and allows a dynamic assessment. However, it is highly user dependent, and the quality of scanners is variable. MRI allows for a detailed overall assessment of the shoulder girdle and gives reliable outcomes. This modality is associated with consistently accurate results and therefore became the gold standard. However, the confined space in MRI scanners is less tolerable for patients. Moreover, MRI is a static type of imaging, and the ever increasing demand on MRI services leads to delays. Because of that, improvements have been made in ultrasound techniques. The development of office-based, 
high-intensity ultrasound and the emergence of specialist musculoskeletal (MSK) sonographers allow for a swift and accurate identification of rotator cuff tears [1]. This has enabled ultrasound to compete with MRI in terms of reliability and efficacy.

Recently, the available evidence has provided guidelines for diagnosing rotator cuff tears. UKUFF was a multi-centre, randomised, controlled trial assessing rotator cuff treatments. It illustrated the beneficial impact of surgical management of these injuries. Accurate diagnosis is therefore key in defining patients who can benefit from interventions [2].

A recent Cochrane review of the subject suggested that US and MRI both performed well with respect to full thickness rotator cuff tears; however, they were less accurate with respect to partial thickness tears [3]. Other smaller scale studies mirror these effects with comparable results between the two modalities. In 2003, Dinnes et al. showed that both imaging techniques could be used with good effect to view full thickness tears, but ultrasound may be better at diagnosing partial thickness tears [4].

The aim of this study is to compare the ability of pre-operative US and MRI in detecting rotator cuff tears with reference to arthroscopic findings as the gold standard for diagnosis rotator cuff tears.

\section{Material and Methods}

We conducted a retrospective analysis of 300 consecutive patients who underwent arthroscopic interventions between October 2013 and October 2015. All operations were performed or supervised by the senior author.

Theatre lists were used to identify patients, and the operating notes were reviewed to obtain arthroscopic diagnoses. Pre-operatively, patients underwent imaging with ultrasound or MRI. These were assessed and reported by consultant radiologists. The presence of a full or partial thickness rotator cuff tear was noted. The imaging reports were then compared against the operative findings. Forty-five patients were excluded from the study (30 due to inaccurate coding, 10 due to unavailability of scan reports, and 5 due to lack of information with respect to the rotator cuff in procedural notes). This left a final cohort of 255 patients that was divided into two groups: 125 patients who had US and 130 patients who had MRI. The US group: $52 \%$ females and $48 \%$ males, average age of 52 years. The MRI group: $53 \%$ females and $47 \%$ males, average age of 54 years. All US examinations were performed by senior MSK radiologists using a high-resolution US machine. MRI scans were performed using 1.5 or 3 Tesla MRI scanners. The US and MRI reporters were not blind to the patient history and examination findings. The surgeon was not blind to the clinical findings and the imaging reports.

The imaging result of each patient was compared with arthroscopic findings, representing the reference standard. Categorical data were compared using the Fischer exact test, rather than $\mathrm{t}$ chi-squared, as each test had at least one group with less than 10 subjects. The MRI and ultrasound groups were compared against intra-operative findings using a linear-weighted Kappa statistic.

A priori power analysis was conducted using the $\mathrm{G}^{*}$ Power statistical software (V 3.1.3 Franz Faul, Universitat Kiel, Germany) [5]. Alpha was set at 0.05 and Beta at 0.2. Cohen's effect size for categorical variables was set at 0.3 , indicating that the calculation would be able to detect a 'medium'-sized difference between the groups [6]. The calculation was adjusted to use three degrees of freedom.

Results of the power calculation indicate that 122 patients were required to achieve $80 \%$ power.

\section{Results}

Arthroscopically, we found that, of the 125 patients in the US group, 61 had a rotator cuff tear (RCT) of any type, and 64 patients had no rotator cuff tears (NT). Out of those 61 patients, 48 had a full thickness cuff tear (FTT), and 13 patients had a partial thickness cuff tear (PTT). In the MRI group, we found that 79 patients had a RCT, 55 patients had a FTT, and 24 patients had a PTT. Fifty-one patients in this group had NT. The mean time between the scan and surgery was 29 weeks for the US group and 25 weeks for the MRI group.

When we compared the arthroscopic results to the US and MRI findings, we found that US was 77\% sensitive and 82.8\% specific in detection of all RCT, with a positive prediction value (PPV) of $81 \%$ and a negative prediction value (NPV) of $79.1 \%$. MRI was more sensitive (89.9\%) but less specific $(66.6 \%)$ in detection of RCT, with PPV of $80.6 \%$ and NPV of $81 \%$. Based on these results, there was no significant difference in detecting RCTs between the two methods $(\mathrm{P}=0.613$ for sensitivity and $\mathrm{P}=1$ for specificity, $\mathrm{PPV}$, and NPV).

In FTTs, we found that US was $77 \%$ sensitive and $90.9 \%$ specific, with PPV of $84 \%$ and NPV of $86.4 \%$. MRI was less sensitive (69\%) and less specific (89.3\%) than US in detecting FTT, with PPV of $82.6 \%$ and NPV of $79.7 \%$. Similarly, these results confirmed that there was no significant difference between US and MRI in detecting FTT, $(\mathrm{P}=0.762$ for sensitivity, $\mathrm{P}=0.791$ for specificity, $\mathrm{P}=1$ for $\mathrm{PPV}$, and $\mathrm{P}=0.302$ for $\mathrm{NPV}$ ).

We found that US was less sensitive in detecting PTT compared to MRI (23\% and 54.1\%, respectively), but this difference was not significant $(\mathrm{P}=0.333)$. In contrast, it was found that US was more specific (90.1\%) than MRI (72.6\%), which was statistically significant $(\mathrm{P}=0.0008)$. PPV was $21.4 \%$ for US and $30.9 \%$ for MRI, with no significant difference $(p=0.73)$. NPV was $90.9 \%$ for US and $87.5 \%$ for MRI, with no significant difference $(\mathrm{p}=0.48)$. Table 1 summarises the above results.

\section{Discussion}

Accurate pre-operative imaging allows for an informed discussion with the patient and an optimal management planning of any shoulder pathology, in particular rotator cuff tears. US and MRI have been in use for a long time as diagnostic tools for rotator cuff pathology. Different 
Table 1. Summary of the results.

\begin{tabular}{|c|c|c|c|c|c|}
\hline & & Sensitivity & Specificity & PPV & NPV \\
\hline \multirow{3}{*}{$\mathrm{RCT}$} & US & $77 \%$ & $82.8 \%$ & $81 \% \%$ & $79.1 \%$ \\
\hline & MRI & $89.8 \%$ & $66.6 \%$ & $80.6 \%$ & $81 \%$ \\
\hline & Pvalue & 0.613 & 1 & 1 & 1 \\
\hline \multirow{3}{*}{ FTT } & US & $77 \%$ & $90.9 \%$ & $84 \%$ & $86.4 \%$ \\
\hline & MRI & $69 \%$ & $89.3 \%$ & $82.6 \%$ & $79.7 \%$ \\
\hline & $P$ value & 0.762 & 0.791 & 1 & 0.302 \\
\hline \multirow{3}{*}{ PTT } & US & $23 \%$ & $90.1 \%$ & $21.4 \%$ & $90.9 \%$ \\
\hline & MRI & $54.1 \%$ & $72.6 \%$ & $30.9 \%$ & $87.5 \%$ \\
\hline & Pvalue & 0.333 & 0.0008 & 0.73 & 0.489 \\
\hline
\end{tabular}

levels of accuracy have been reported with different levels of evidence for full and partial thickness tears $[3,4,7,8]$. De Jesus et al., in a meta-analysis, reported of higher sensitivity and specificity of MRI arthrography over US and MRI [9]. However, a more recent Cochrane review reported no significant difference between US and MRI in detection of rotator cuff tears with sensitivity of $91 \%$ and $98 \%$, and with specificity of $79 \%$ and $85 \%$, respectively $(\mathrm{P}=0.13)$ [8]. These results are comparable to the results in our series which showed no significant difference, although the sensitivity values were lower than those in the previously mentioned studies. Rutten et al., based on a retrospective study, showed no statistical difference between US and MRI for detection of rotator cuff tears [10]. In our series, we found no significant difference in all measured parameters with only one exception. We found that US is significantly more specific in detection of PTT, which means that US is more capable of excluding false positive results compared to MRI. This could be also secondary to the fact that small intra-substance cuff tears that are detected by MRI could not be seen intra-operatively, which is also operator dependent. However, the difference in accuracy in PTT diagnosis, although statistically significantly, may not be clinically apparent, since the first-line treatment of PTT is non-operative [11].

In addition to the diagnostic value, some other factors contribute to the selection of appropriate imaging examination. US is more tolerated by patients, more available, more cost-effective, and with shorter waiting time. However, US is operator dependent. In our series, all US examinations were performed by radiologists, although US could be also be performed by trained shoulder surgeons with a similar level of accuracy [12].

There were a few limitations to our study. A retrospective study can be associated with a selection bias among patients who had already had surgery. Therefore, positive findings are more likely to be obtained. Scan reports and surgeons were not blinded to clinical findings, which could lead to verification bias. It would have been more accurate, if all procedures had been performed by the same operator. However, that was difficult and could have affected the number of patients in the cohort.

\section{Conclusions}

US and MRI have similar abilities to diagnose RCT of any sort or FTT. Diagnosing FTT with US is more specific than with MRI. In our institute, we recommend US as an investigation of choice for diagnosing rotator cuff tears.

\section{Conflict of interest}

All authors declare that they have no conflict of interest.

\section{Acknowledgment}

We would like to thank Edward Dawe and the ORKSS Collaborative for their support of this study.

\section{References:}

1.Iannotti JP, Coccone J, Buss DD et al: Accuracy of office-based ultrasonography of the shoulder for the diagnosis of rotator cuff tears. J Bone Joint Surg Am, 2005; 87(6): 1305-11

2.Carr AJ, Cooper CD, Campbell MK et al: Clinical effectiveness and cost-effectiveness of open and arthroscopic rotator cuff repair [the UK Rotator Cuff Surgery (UKUFF) randomised trial]. Health Technol Assess, 2015; 19(80): 1-218

3.Lenza M, Buchbinder R, Tawoingi $Y$ et al: Magnetic resonance imaging, magnetic resonance arthrography and ultrasonography for assessing rotator cuff tears in people with shoulder pain for whom surgery is being considered. Cochrane Database Syst Rev, 2013; (9): CD009020
4.Dinnes J, Loveman E, Mclntyre L, Waugh N: The effectiveness of diagnostic tests for the assessment of shoulder pain due to soft tissue disorder: A systematic review. Health Technol Assess, 2003; 7(29): iii, $1-166$

5.Erdfelder E, Faul F, Buchner A, Lang AG: A G*POWER3: A general power analysis program. Behaviour Research Methods Instruments \& Computers, 2006; 28: 1-11

6.Cohen J: Statistical power for the behavioural sciences, $2^{\text {nd }}$ ed., Erlbaum, Hillsdale NJ, 1988

7.Smith TO, Back T, Toms A, Hing CB: Diagnostic accuracy of ultrasound for rotator cuff tears in adults: A systematic review and matsanalysis. Clin Radiol, 2011; 66: 1036-48 
8.Modi CS, Smith CD, Ho K et al: Accuracy of high-resolution ultrasonography in the diagnosis of articular side partial thickness rotator cuff tears. Shoulder and Elbow, 2010; 2: 267-70

9.De Jesus JO, Parker L, Frangos AJ, Nazarian LN: Accuracy of MRI, MRI arthrography and ultrasound in the diagnosis of rotator cuff tears: A meta-analysis. Am J Roentgenol, 2009; 192(6): 1701-7

10.Rutten MJ, Spaargaren GJ, van Loon T et al: Detection of rotator cuff tears: Value of MRI following Ultrasound. Eur Radiol, 2010; 20: $450-57$
11.Lambers Heerspink FO, Dorrestijn O, van Raay JJ, Diercks RL: Specific patient related prognostic factors for rotator cuff repair: A systemic review. J Shoulder Elbow Surg, 2014; 23: 1073-80

12.Jeyam M, Funk L, Harris J: Are shoulder surgeons any good at diagnosing rotator cuff tears using ultrasound? A comparative analysis of surgeon vs. radiologist. Int J Shoulder Surg, 2008; 2(1): 4-6 